\title{
Correlation Between Human Aesthetic Judgement and Spatial Complexity Measure
}

\author{
Mohammad Ali Javaheri Javid, Tim Blackwell, Robert Zimmer Mohammad \\ Majid al-Rifaie \\ m.javaheri, t.blackwell,r.zimmer,m.majid@gold.ac.uk \\ Department of Computing \\ Goldsmiths, University of London \\ London SE14 6NW, UK
}

\begin{abstract}
The quantitative evaluation of order and complexity conforming with human intuitive perception has been at the core of computational notions of aesthetics. Informational theories of aesthetics have taken advantage of entropy in measuring order and complexity of stimuli in relation to their aesthetic value. However entropy fails to discriminate structurally different patterns in a $2 \mathrm{D}$ plane. This paper investigates a computational measure of complexity, which is then compared to a results from a previous experimental study on human aesthetic perception in the visual domain. The model is based on the information gain from specifying the spacial distribution of pixels and their uniformity and nonuniformity in an image. The results of the experiments demonstrate the presence of correlations between a spatial complexity measure and the way in which humans are believed to aesthetically appreciate asymmetry. However the experiments failed to provide a significant correlation between the measure and aesthetic judgements of symmetrical images.
\end{abstract}

Keywords: human aesthetic judgements, spatial complexity, information theory, symmetry, complexity

\section{Introduction}

The advent of computers and subsequent advances in hardware and software, especially the development of tools for interactively creating graphical contents, have turned purely calculating machines into full-fledged artistic tools, as expressive as a brush and canvas. As noted by Michael Noll, one of the early pioneers of computer art, "in the computer, man has created not just an inanimate tool but an intellectual and active creative partner that, when fully exploited, could be used to produce wholly new art forms and possibly new aesthetic experiences" [32, p.89].

Biologically inspired generative tools, especially those utilising evolutionary methods, have been contributed to the creation of various computer generated art with aesthetic qualities. The Biomorphs of Dawkins [12], Mutators of Latham [24], and Virtual Creatures of Sims [36] are classic examples of evolutionary art. 
An open problem in evolutionary art is to automate aesthetic judgements so that only images of high aesthetic quality are generated [10]. However there are a number of challenges when dealing with evolutionary methods [28]. Therefore, the development of a model of aesthetic judgement model is one of the major challenges in evolutionary art [29] and an essential step for the creation of an autonomous system [27] where both of the generation and evaluation process are integrated.

This paper is organised as follows. In Section 2 the relationship between aesthetics and complexity is examined. The notion of complexity from the perspective of Shannon information theory is analysed and its influence on informational theories of aesthetics is discussed. In section 3 we discuss the drawback of entropic approaches for aesthetic evaluation purposes. An in-depth analysis of entropic measure for $2 \mathrm{D}$ patterns with examples is provided. In the framework of the objectives of this study, a spatial complexity spectrum is formulated and the potential of information gain as a spatial complexity measure is discussed. In section 4, we provide details of experiments and their results on the correlation of a spatial complexity model with human aesthetic judgements. In section 5 , a discussion and a summary of findings is provided.

\section{Informational Theories of Aesthetics}

Aesthetics has traditionally been a branch of philosophy dealing with the nature of beauty in its synthetic forms (i.e. artworks) and its natural forms (e.g. the beauty of a sunset). Computational aesthetics is concerned with the development of computational methods to make human-like aesthetic judgements. The main focus is on developing aesthetic measures as functions which compute the aesthetic value of an object [13]. There a sizeable body of literature on various computational approaches to aesthetics $[18,19]$. Our review mainly spans models derived from Birkhoff's aesthetic measure and information theory.

Aesthetic judgements have long been hypothesised to be influenced by the degree of order in a stimulus (i.e. symmetry) and the complexity of the stimulus. Birkhoff proposed a mathematical aesthetic measure by arguing that the measure of aesthetic quality $(M)$ (Eq. 1) is in direct relation to the degree of order $(O)$ and in reverse relation to the complexity $(C)$ of an object $[9]$,

$$
M=\frac{O}{C} .
$$

The validity of Birkhoff's model, and his definition of order and complexity, has been challenged by empirical studies [39]. Eysenck conducted a series of experiments on Birkhoff's model and suggested that a better expression of aesthetic evaluation function should consider a direct relation to stimulus complexity rather than an inverse relation $(M=O \times C)[15,16,14]$. Although the validity of Birkhoff's approach in penalising complexity has been challenged by empirical studies, the notion of order and complexity and objective methods to quantify them remains a prominent concern in aesthetic evaluation functions. 
Information theory addresses the problem of a reliable communication over an unreliable channel [35]. Entropy is the core of this theory [11]. Let $\mathcal{X}$ be discrete alphabet, $X$ a discrete random variable, $x \in \mathcal{X}$ a particular value of $X$ and $P(x)$ the probability of $x$. Then the entropy, $H(X)$, is:

$$
H(X)=-\sum_{x \in \mathcal{X}} P(x) \log _{2} P(x) .
$$

The quantity $H$ is the average uncertainty in bits, $\log _{2}\left(\frac{1}{p}\right)$ associated with $X$. Entropy can also be interpreted as the average amount of information needed to describe $X$. The value of entropy is always non-negative and reaches its maximum for the uniform distribution, $\log _{2}(|\mathcal{X}|)$ :

$$
0 \leqslant H \leqslant \log _{2}(|\mathcal{X}|) .
$$

The lower bound of relation (3) corresponds to a deterministic variable (no uncertainty) and the upper bound corresponds to a maximum uncertainty associated with the random variable. Entropy is regarded as a measure of order and complexity. A low entropy implies low uncertainty so the message is highly predictable, ordered and less complex. And high entropy implies a high uncertainty, less predictability, highly disordered and complex. These interpretations of entropy provided quantitative means to measure order and complexity of objects in relation to their aesthetic value and consequently contributed to the development informational aesthetics, an information-theoretic interpretation of aesthetics.

Moles [30], Bense [7, 6, 8] and Arnheim [2, 3, 4] were pioneers of the application of entropy to quantify order and complexity in Birkhoff's formula by adapting statistical measure of information in aesthetic objects. Bense argued that aesthetic objects are "vehicles of aesthetical information" where statistical information can quantify the aesthetical information of objects [7]. His informational aesthetics has three basic assumptions. (1) Objects are material carriers of aesthetic state, and such aesthetic states are independent of subjective observers. (2) A particular kind of information is conveyed by the aesthetic state of the object (or process) as aesthetic information and (3) objective measure of aesthetic objects is in relation with degree of order and complexity in an object [31].

Herbert Franke put forward a cybernetic aesthetics based on aesthetic perception. He made a distinction between the amount of information being stored and the rate of information flowing through a channel as information flow measured in bits/sec [17]. His theory is based on psychological experiments which suggested that conscious working memory can not take more than 16 bits/sec of visual information. Then he argued that artists should provide a flow of information of about $16 \mathrm{bits} / \mathrm{sec}$ for works of art to be perceived as beautiful and harmonious.

Staudek in his multi-criteria approach (informational and structural) as exact aesthetics to Birkhoff's measure applied information flow $I^{\prime}$ by defining it as a 
measure assessing principal information transmission qualities in time. He used $16 \mathrm{bits} / \mathrm{sec}$ reference as channel capacity $C_{r}=16 \mathrm{bits} / \mathrm{sec}$ and a time reference of 8 seconds $\left(t_{r}=8 \mathrm{~s}\right)$ to argue that artefacts with $I>128$ bits will not fit into the conscious working memory for absorbing the whole aesthetic message [37].

Machado and Cardoso proposed a model based on Birkhoff's approach as the ratio of image complexity to processing complexity by arguing that images with high visual complexity, are processed easily so they have highest aesthetic value [26]. Adapting Bense's informational aesthetics to different approaches of the concepts of order and complexity in an image, three measures based on Kolmogorov complexity [25], Shannon entropy (for RGB channels) and Zurek's physical entropy [40] were introduced. Then the measures were applied to analyse aesthetic values of several paintings (Mondrian, Pollock, and van Gogh) [34, 33].

\section{Spatial Complexity Measure}

Despite the dominance of entropy as a measure of order and complexity, it fails to capture structural characteristics of $2 \mathrm{D}$ patterns. The main reason for this drawback is that entropy is a function of the distribution of the symbols, and not on their spatial arrangement [23]. Consequently any model derived from information theory will inherently suffer from this drawback.

Considering our intuitive perception of complexity and structural characteristics of $2 \mathrm{D}$ patterns, a complexity measure must be bounded by two extreme points of complete order and disorder. It is reasonable to assume that regular structures, irregular structures and structureless patterns lie along between these extremes, as illustrated in Fig. 1.

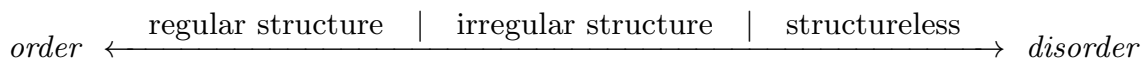

Fig. 1: The spectrum of spatial complexity.

A complete regular structure is a pattern of high symmetry, an irregular structure is a pattern with some sort of structure but not as regular as a fully symmetrical pattern and finally a structureless pattern is a random arrangement of elements [22]. A measure introduced in [5, 38, 1] and known as information gain, has been proposed as a means of characterising the complexity of dynamical systems and of $2 \mathrm{D}$ patterns. It measures the amount of information gained in bits when specifying the value, $x$, of a random variable $X$ given knowledge of the value, $y$, of another random variable $Y$,

$$
G_{x, y}=-\log _{2} P(x \mid y) .
$$

$P(x \mid y)$ is the conditional probability of a state $x$ conditioned on the state $y$. Then the mean information gain (MIG), $\bar{G}_{X, Y}$, is the average amount of information 
gain from the description of the all possible states of $Y$ :

$$
\bar{G}_{X, Y}=\sum_{x, y} P(x, y) G_{x, y}=-\sum_{x, y} P(x, y) \log _{2} P(x \mid y)
$$

where $P(x, y)$ is the joint probability, $\operatorname{prob}(X=x, Y=y) . \bar{G}$ is also known as the conditional entropy, $H(X \mid Y)$ [11]. Conditional entropy is the reduction in uncertainty of the joint distribution of $X$ and $Y$ given knowledge of $Y, H(X \mid Y)=$ $H(X, Y)-H(Y)$. The lower and upper bounds of $\bar{G}_{X, Y}$ are

$$
0 \leqslant \bar{G}_{X, Y} \leqslant \log _{2}|\mathcal{X}| .
$$

The structural characteristics of a $2 \mathrm{D}$ image are determined by the spatial distribution and state (i.e. colour) of individual pixels. In terms of the state of pixels they can be either with a uniform state relation (same colours) or nonuniform state relation (different colours) with their neighbouring pixels. In order to apply $G$ to an image, the following definitions are needed:

- $L$ is a finite lattice of pixels $(i, j)$.

- $S=\{1,2, \ldots, k\}$ is set of states. Each pixels $(i, j)$ in $L$ has a state $s \in S$.

- $N$ is neighbourhood, as specified by a set of lattice vectors $\left\{e_{a}\right\}, a=$ $1,2, \ldots, N$. The neighbourhood of pixel $r=(i, j)$ is $\left\{r+e_{1}, r+e_{2}, \ldots, r+e_{N}\right\}$. With an economy of notation, the pixels in the neighbourhood of $(i, j)$ can be numbered from 1 to $N$; the neighbourhood states of $(i, j)$ can therefore be denoted $\left(s_{1}, s_{2}, \ldots, s_{N}\right)$. An eight-cell neighbourhood $\{( \pm 1,0),(0, \pm 1),( \pm 1, \pm 1)\}$ is considered for a pixel's relation to its neighbouring pixels. The relative positions for non-edge pixels, since they do not have neighbouring pixels, is given by matrix $M$ :

$$
M=\left[\begin{array}{ccc}
(i-1, j+1) & (i, j+1) & (i+1, j+1) \\
(i-1, j) & (i, j) & (i+1, j) \\
(i-1, j-1) & (i, j-1) & (i+1, j-1)
\end{array}\right] .
$$

For an image, $\bar{G}$ can be calculated by considering the distribution of pixel colours over pairs of pixels $r, s$,

$$
\bar{G}_{r, s}=-\sum_{s_{r}, s_{s}} P\left(s_{r}, s_{s}\right) \log _{2} P\left(s_{r}, s_{s}\right)
$$

where $s_{r}, s_{s}$ are the states at $r$ and $s$. Since $|S|=N, \bar{G}_{r, s}$ is a value in $[0, N]$ (details of calculations for a sample pattern are provided in appendix). The vertical, horizontal, primary diagonal $(\backslash)$ and secondary diagonal $(/)$ neighbouring pairs provide eight $\bar{G} s ; \bar{G}_{(i, j),(i-1, j+1)}, \bar{G}_{(i, j),(i, j+1)}, \bar{G}_{(i, j),(i+1, j+1)}, \bar{G}_{(i, j),(i-1, j)}$, $\bar{G}_{(i, j),(i+1, j)}, \bar{G}_{(i, j),(i-1, j-1)}, \bar{G}_{(i, j),(i, j-1)}$ and $\bar{G}_{(i, j),(i+1, j-1)}$. Correlations between pixels on opposing lattice edges are not considered. The result of this edge condition is that $G_{i+1, j}$ is not necessarily equal to $\bar{G}_{i-1, j}$.

Fig. 2 illustrates the advantages of $\bar{G}$ over $H$ in discriminating structurally different patterns where the elements are equally probable $\left(P\left(s_{r}, s_{s}\right)=\frac{1}{108}\right)$. 
Fig. 2a is completely symmetrical, Fig. $2 \mathrm{~b}$ is partially structured and Fig. 2c is a structureless and random pattern. The calculations have been performed for each element of images having a uniform and non-uniform colours in their relative spatial positions for three possible colours $(S=\{$ lightgrey, grey, black $\})$ along with $\bar{G}$, and $\mu(\bar{G})$, the mean of the eight directional $G$ 's. As is evident, $H$ is identical for these structurally different patterns, however, $\bar{G}$ and $\mu(\bar{G})$ reflect the order and complexity of patterns due to the spatial arrangements of composing elements. Fig. 2 clearly demonstrates the drawbacks of entropy to discriminate structurally different $2 \mathrm{D}$ patterns. In other words, entropy is invariant to the spatial arrangement of the composing elements.

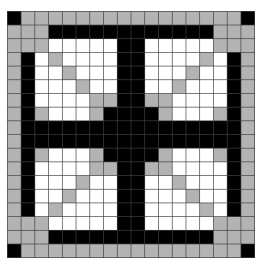

(a)

$$
\begin{gathered}
\bar{G}_{i, j+1}=1.32480 \\
\bar{G}_{i, j-1}=1.32480 \\
\bar{G}_{i-1, j}=1.32480 \\
\bar{G}_{i+1, j}=1.32480 \\
\bar{G}_{i-1, j+1}=1.45033 \\
\bar{G}_{i+1, j-1}=1.45033 \\
\bar{G}_{i+1, j+1}=1.45033 \\
\bar{G}_{i-1, j-1}=1.45033
\end{gathered}
$$

$$
\mu(G)=1.38756
$$

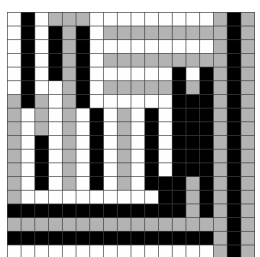

(b)

$$
H=1.58496
$$

$$
\begin{gathered}
\bar{G}_{i, j+1}=1.32515 \\
\bar{G}_{i, j-1}=1.32703 \\
\bar{G}_{i-1, j}=1.54180 \\
\bar{G}_{i+1, j}=1.54630 \\
\bar{G}_{i-1, j+1}=1.36769 \\
\bar{G}_{i+1, j-1}=1.36670 \\
\bar{G}_{i+1, j+1}=1.38744 \\
\bar{G}_{i-1, j-1}=1.38962
\end{gathered}
$$

$\mu(G)=1.40646$

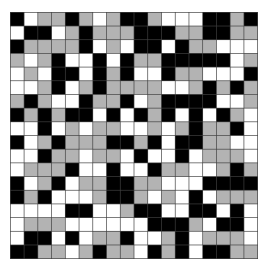

(c)

$$
H=1.58496
$$

$$
\begin{gathered}
\bar{G}_{i, j+1}=1.58181 \\
\bar{G}_{i, j-1}=1.58209 \\
\bar{G}_{i-1, j}=1.57696 \\
\bar{G}_{i+1, j}=1.57668 \\
\bar{G}_{i-1, j+1}=1.56727 \\
\bar{G}_{i+1, j-1}=1.56712 \\
\bar{G}_{i+1, j+1}=1.57688 \\
\bar{G}_{i-1, j-1}=1.57657
\end{gathered}
$$

$\mu(G)=1.57567$

Fig. 2: Measures of $H, \bar{G} s$ and $\mu(G)$ for structurally different patterns with equally probable distribution of elements.

\section{Analysis}

The purpose of this section compare $\mu(G)$ for twelve patterns with an empirical aesthetic ranking.

Twelve experimental stimuli were adapted from an empirical study of human aesthetic judgement [21]. Jacobsen [21] reports on an empirical trial of human aesthetic judgement. Fifty-five young adults (15 males) participated in the experiment for course credit or partial fulfilment of course requirements. All were first or second year psychology students at the University of Leipzig. None 
of them had received professional training in the fine arts or participated in a similar experiment before. Participants reported normal or corrected-to-normal visual acuity. Subjects were asked to evaluate images from two groups; a group of asymmetrical images and a group of symmetrical images with at least one axis of reflection symmetry. The images consisted of a solid black circle showing a centred, quadratic, rhombic cut-out.

Twelve of these images were scaled to $151 \times 151$ pixels $(S=\{$ white, black $\})$ and the black circular background was replaced by a square in order to reduce aliasing errors. The directional information gains, Eq. 8, and the mean gain, $\mu(G)$, were calculated. The results are detailed in Fig. 4 and Fig. 6 .

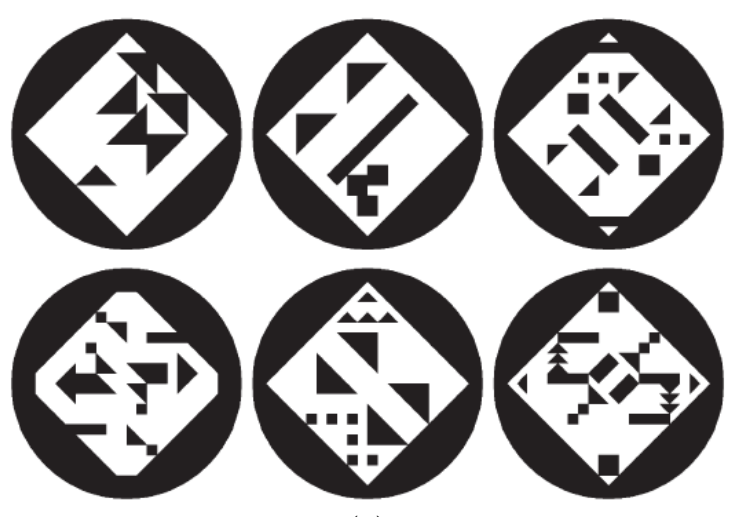

(a)

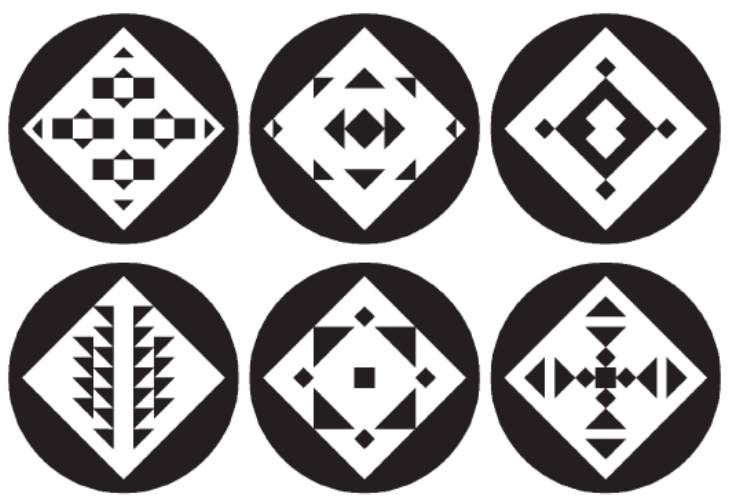

(b)

Fig. 3: Stimulus examples from [20]. The patterns in (a) are not symmetric, ranging from not beautiful (as judged in the trials) to beautiful (from top left for bottom right) and the patterns in (b) are symmetric, ranging also from not beautiful to beautiful (from top left for bottom right) 


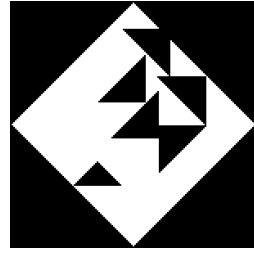

(1)

$\bar{G}_{i, j+1}=0.18216$

$\bar{G}_{i, j-1}=0.18216$

$\bar{G}_{i-1, j}=0.17310$

$\bar{G}_{i+1, j}=0.17310$

$\bar{G}_{i-1, j+1}=0.20089$

$\bar{G}_{i+1, j-1}=0.20089$

$\bar{G}_{i+1, j+1}=0.21633$

$\bar{G}_{i-1, j-1}=0.21633$

$\mu(G)=0.19312$

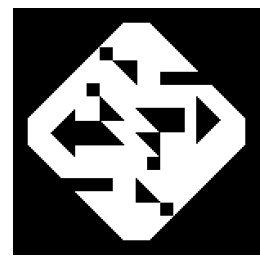

(4)

$\bar{G}_{i, j+1}=0.20093$

$\bar{G}_{i, j-1}=0.20093$

$\bar{G}_{i-1, j}=0.17433$

$\bar{G}_{i+1, j}=0.17433$

$\bar{G}_{i-1, j+1}=0.25834$

$\bar{G}_{i+1, j-1}=0.25834$

$\bar{G}_{i+1, j+1}=0.23194$

$\bar{G}_{i-1, j-1}=0.23194$

$$
\mu(G)=0.21639
$$

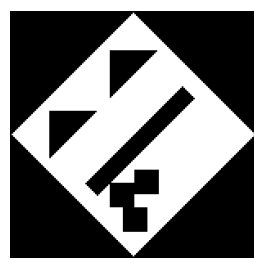

(2)

$\bar{G}_{i, j+1}=0.18034$

$\bar{G}_{i, j-1}=0.18034$

$\bar{G}_{i-1, j}=0.18034$

$\bar{G}_{i+1, j}=0.18034$

$\bar{G}_{i-1, j+1}=0.17402$

$\bar{G}_{i+1, j-1}=0.17402$

$\bar{G}_{i+1, j+1}=0.24146$

$\bar{G}_{i-1, j-1}=0.24146$

$\mu(G)=0.19404$

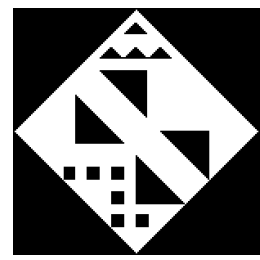

(5)

$\bar{G}_{i, j+1}=0.20752$

$\bar{G}_{i, j-1}=0.20752$

$\bar{G}_{i-1, j}=0.19412$

$\bar{G}_{i+1, j}=0.19412$

$\bar{G}_{i-1, j+1}=0.27130$

$\bar{G}_{i+1, j-1}=0.27130$

$\bar{G}_{i+1, j+1}=0.22552$

$\bar{G}_{i-1, j-1}=0.22552$

$\mu(G)=0.22462$

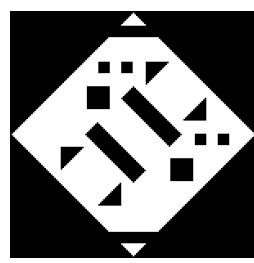

(3)

$\bar{G}_{i, j+1}=0.20431$

$\bar{G}_{i, j-1}=0.20431$

$\bar{G}_{i-1, j}=0.18502$

$\bar{G}_{i+1, j}=0.18502$

$\bar{G}_{i-1, j+1}=0.24456$

$\bar{G}_{i+1, j-1}=0.24456$

$\bar{G}_{i+1, j+1}=0.22990$

$\bar{G}_{i-1, j-1}=0.22990$

$\mu(G)=0.21595$

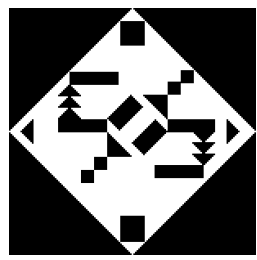

(6)

$\bar{G}_{i, j+1}=0.24608$

$\bar{G}_{i, j-1}=0.24608$

$\bar{G}_{i-1, j}=0.21380$

$\bar{G}_{i+1, j}=0.21380$

$\bar{G}_{i-1, j+1}=0.28628$

$\bar{G}_{i+1, j-1}=0.28628$

$\bar{G}_{i+1, j+1}=0.28925$

$\bar{G}_{i-1, j-1}=0.28925$

$$
\mu(G)=0.25885
$$

Fig. 4: The measurement of $G s$ for asymmetrical stimuli in bits.

The relationship between aesthetic judgements and $\mu(G)$ for asymmetrical stimuli are shown in Fig. 5. The analysis shows a strong positive correlation between aesthetic judgements and $\mu(G)(r=0.9327, y=0.012 x+0.175)$. This indicates that information gain can be directly linked to the human aesthetic judgements of asymmetrical patterns. 


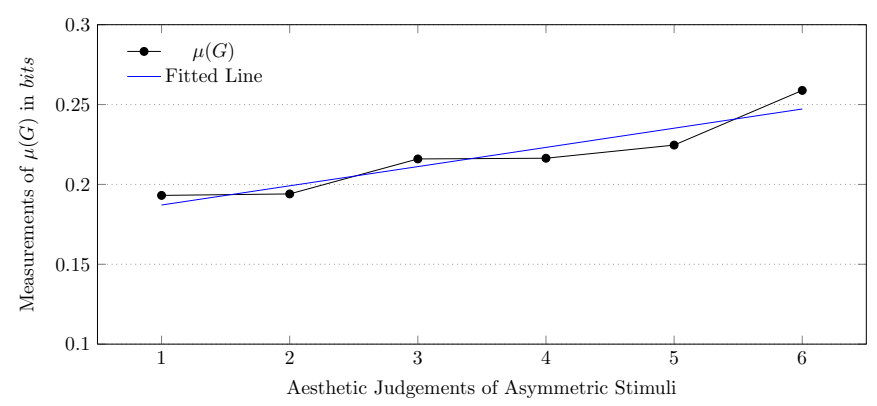

Fig. 5: The measurements of $\mu(G)$ for asymmetrical stimuli.

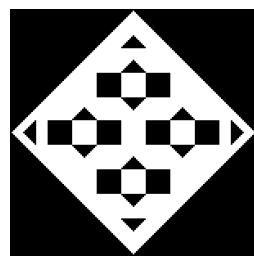

(1)

$\bar{G}_{i, j+1}=0.23315$

$\bar{G}_{i, j-1}=0.23315$

$\bar{G}_{i-1, j}=0.20992$

$\bar{G}_{i+1, j}=0.20992$

$\bar{G}_{i-1, j+1}=0.28055$

$\bar{G}_{i+1, j-1}=0.28055$

$\bar{G}_{i+1, j+1}=0.28055$

$\bar{G}_{i-1, j-1}=0.28055$

$\mu(G)=0.25104$

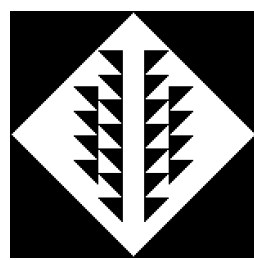

(4)

$\bar{G}_{i, j+1}=0.24350$

$\bar{G}_{i, j-1}=0.24350$

$\bar{G}_{i-1, j}=0.24746$

$\bar{G}_{i+1, j}=0.24746$

$\bar{G}_{i-1, j+1}=0.30361$

$\bar{G}_{i+1, j-1}=0.30361$

$\bar{G}_{i+1, j+1}=0.30361$

$\bar{G}_{i-1, j-1}=0.30361$

$$
\mu(G)=0.27455
$$

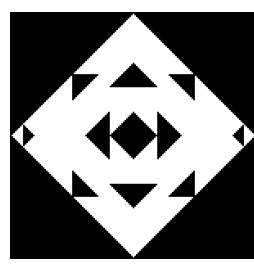

(2)

$\bar{G}_{i, j+1}=0.19365$

$\bar{G}_{i, j-1}=0.19365$

$\bar{G}_{i-1, j}=0.19804$

$\bar{G}_{i+1, j}=0.19804$

$\bar{G}_{i-1, j+1}=0.22226$

$\bar{G}_{i+1, j-1}=0.22226$

$\bar{G}_{i+1, j+1}=0.22226$

$\bar{G}_{i-1, j-1}=0.22226$

$\mu(G)=0.20905$

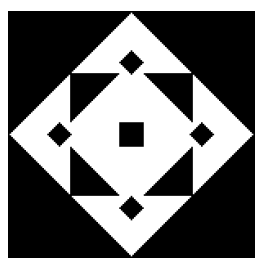

(5)

$\bar{G}_{i, j+1}=0.19313$

$\bar{G}_{i, j-1}=0.19313$

$\bar{G}_{i-1, j}=0.19313$

$\bar{G}_{i+1, j}=0.19313$

$\bar{G}_{i-1, j+1}=0.22414$

$\bar{G}_{i+1, j-1}=0.22414$

$\bar{G}_{i+1, j+1}=0.22414$

$\bar{G}_{i-1, j-1}=0.22414$

$\mu(G)=0.20864$

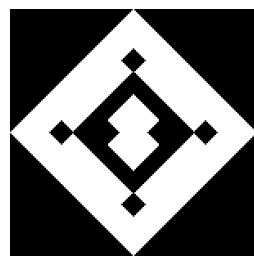

(3)

$\bar{G}_{i, j+1}=0.18736$

$\bar{G}_{i, j-1}=0.18736$

$\bar{G}_{i-1, j}=0.18825$

$\bar{G}_{i+1, j}=0.18825$

$\bar{G}_{i-1, j+1}=0.18882$

$\bar{G}_{i+1, j-1}=0.18882$

$\bar{G}_{i+1, j+1}=0.18882$

$\bar{G}_{i-1, j-1}=0.18882$

$\mu(G)=0.18831$

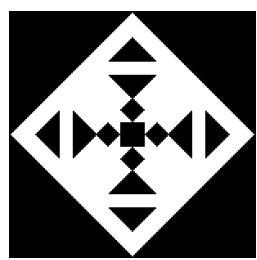

(6)

$\bar{G}_{i, j+1}=0.21635$

$\bar{G}_{i, j-1}=0.21635$

$\bar{G}_{i-1, j}=0.21635$

$\bar{G}_{i+1, j}=0.21635$

$\bar{G}_{i-1, j+1}=0.24543$

$\bar{G}_{i+1, j-1}=0.24543$

$\bar{G}_{i+1, j+1}=0.24543$

$\bar{G}_{i-1, j-1}=0.24543$

$\mu(G)=0.23089$

Fig. 6: The measurement of Gs for symmetrical stimuli in bits. 


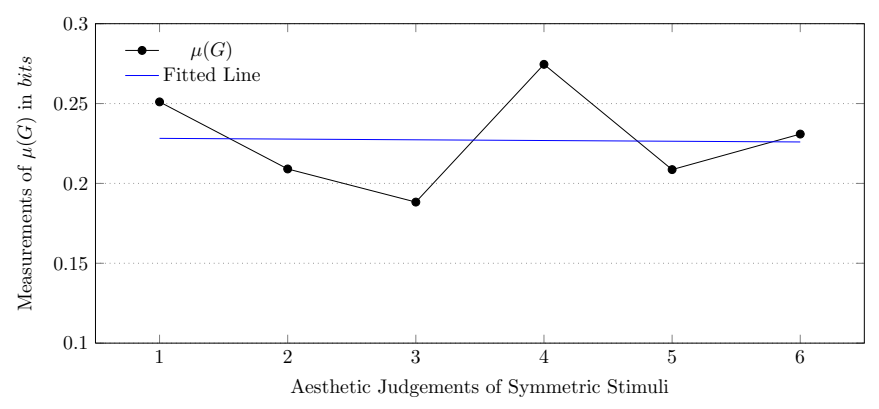

Fig. 7: The measurements of $\mu(G)$ for symmetrical stimuli.

The Pearson correlation coefficient $(r)$ and regression analysis for symmetrical stimuli (Fig. 7) shows no significant correlation between aesthetic judgements and $\mu(G)(r=-0.0266, y=0.229)$. However the images differ in their degree of reflection symmetry. Images 1,2 and 3 have two axes of symmetry, image 4 has a single axes and 5 and 6 have four axes. This mixture of degrees of symmetry might account for the ambiguous results.

\section{Conclusions}

One of the major challenges of evolutionary art and computational notions of aesthetics is the development of a quantitative model which conforms with human intuitive perception. Informational theories of aesthetics based the measurements of entropy have failed to discriminate structurally different patterns in a $2 \mathrm{D}$ plane.

In this work, we investigated information gain as a spatial complexity measure. This measure which takes into account correlations between pixels and can discriminate between structurally different patterns.

This paper reports on the analysis of two different types of stimuli (symmetrical and asymmetrical) which were adapted from an experimental study on human aesthetic perception in the visual domain. The analysis suggest a link between information gain and aesthetic adjustments, in the case of asymmetrical patterns. However, the analysis did not show and link between information gain and empirical aesthetic judgement in the case of patterns with reflection symmetry. It is conjectured that having different orders of reflection symmetry has contributed to this negative finding.

\section{Acknowledgements}

We are grateful to Thomas Jacobsen of Helmut Schmidt University for granting permission to use his experimental stimuli. 


\section{Appendix}

In this example the pattern is composed of two different colours $S=\{$ white, black $\}$ where the set of permutations with repetition is $\{w w, w b, b b, b w\}$. Considering the mean information gain (Eq. 8) and given the matrix $M$ (Eq. 7), the calculations can be performed as follows:

$$
\begin{aligned}
& \square \\
& \text { white - white black - black } \\
& P\left(w, w_{(i, j+1)}\right)=\frac{5}{6} \quad P\left(b, b_{(i, j+1)}\right)=\frac{1}{6} \\
& P\left(w \mid w_{(i, j+1)}\right)=\frac{4}{5} \quad P\left(b \mid b_{(i, j+1)}\right)=\frac{1}{1} \\
& P\left(w, w_{(i, j+1)}\right)=\frac{5}{6} \times \frac{4}{5}=\frac{2}{3} \quad P\left(b, b_{(i, j+1)}\right)=\frac{1}{6} \times \frac{1}{1}=\frac{1}{6} \\
& G\left(w, w_{(i, j+1)}\right)=\frac{2}{3} \log _{2} P\left(\frac{4}{5}\right) \quad G\left(b, b_{(i, j+1)}\right)=\frac{1}{6} \log _{2} P(1) \\
& G\left(w, w_{(i, j+1)}\right)=0.2146 \text { bits } G\left(b, b_{(i, j+1)}\right)=0 \text { bits } \\
& \text { white - black } \\
& \text { black - white } \\
& P\left(w, b_{(i, j+1)}\right)=\frac{5}{6} \quad P\left(b, b_{(i, j+1)}\right)=\frac{1}{6} \\
& P\left(w \mid b_{(j+1)}\right)=\frac{1}{5} \quad P\left(b \mid w_{(i, j+1)}\right)=\frac{0}{1} \\
& P\left(w, b_{(i, j+1)}\right)=\frac{5}{6} \times \frac{1}{5}=\frac{1}{6} \quad P\left(b, w_{(i, j+1)}\right)=\frac{1}{6} \times 0 \\
& \bar{G}\left(w, b_{(i, j+1)}\right)=\frac{1}{6} \log _{2} P \frac{1}{5} \quad G\left(b, w_{(i, j+1)}\right)=0 \text { bits } \\
& \bar{G}\left(w, b_{(i, j+1)}\right)=0.3869 \text { bits } \\
& \bar{G}=G\left(w, w_{(i, j+1)}\right)+G\left(w, b_{(i, j+1)}\right)+G\left(b, b_{(i, j+1)}\right)+G\left(b, w_{(i, j+1)}\right) \\
& \bar{G}=0.6016 \text { bits }
\end{aligned}
$$

In white - white case $G$ measures the uniformity and spatial property where $P\left(w, w_{(i, j+1)}\right)$ is the joint probability that a pixel is white and it has a neighbouring pixel at its $(i, j+1)$ position, $P\left(w \mid w_{(i, j+1)}\right)$ is the conditional probability of a pixel is white given that it has white neighbouring pixel at its $(i, j+1)$ position, $P\left(w, w_{(i, j+1)}\right)$ is the joint probability that a pixel is white and it has neighbouring pixel at its $(i, j+1)$ position, $G\left(w, w_{(i, j+1)}\right)$ is information gain in bits from specifying a white pixel where it has a white neighbouring pixel at its $(i, j+1)$ position. The same calculations are performed for the rest of cases; black-black, white-black and black-white. 


\section{Bibliography}

[1] Andrienko, Yu. A., Brilliantov, N. V., Kurths, J.: Complexity of twodimensional patterns. Eur. Phys. J. B 15(3), 539-546 (2000)

[2] Arnheim, R.: Art and visual perception: A psychology of the creative eye. Univ of California Press (1954)

[3] Arnheim, R.: Towards a psychology of art/entropy and art an essay on disorder and order. The Regents of the University of California (1966)

[4] Arnheim, R.: Visual thinking. Univ of California Press (1969)

[5] Bates, J.E., Shepard, H.K.: Measuring complexity using information fluctuation. Physics Letters A 172(6), 416-425 (1993)

[6] Bense, M., Nee, G.: Computer grafik. In: Bense, M., Walther, E. (eds.) Edition Rot, vol. 19. Walther, Stuttgart (1965)

[7] Bense, M.: Aestetica: Programmierung des Schönen, allgemeine Texttheorie und Textästhetik [Aesthetica : Programming of beauty, general text theory and aesthetics]. Agis-Verlag (1960)

[8] Bense, M.: Kleine abstrakte ästhetik [small abstract aesthetics], Edition Rot, vol. 38. E. Walther (March 1969)

[9] Birkhoff, G.: Aesthetic Measure. Harvard University Press (1933)

[10] Ciesielski, V., Barile, P., Trist, K.: Finding image features associated with high aesthetic value by machine learning. In: Penousal, M., James, M., Adrian, C. (eds.) Evolutionary and Biologically Inspired Music, Sound, Art and Design, Lecture Notes in Computer Science, vol. 7834, pp. 47-58. Springer Berlin Heidelberg (2013)

[11] Cover, T.M., Thomas, J.A.: Elements of Information Theory (Wiley Series in Telecommunications and Signal Processing). Wiley-Interscience (2006)

[12] Dawkins, R.: The blind watchmaker. W. W. Norton, New York (1986)

[13] den Heijer, E., Eiben, A.E.: Comparing Aesthetic Measures for Evolutionary Art. In: Di Chio, C., Brabazon, A., Di Caro, G.A., Ebner, M., Farooq, M., Fink, A., Grahl, J., Greenfield, G., Machado, P., O’Neill, M., Tarantino, E., Urquhart, N. (eds.) EvoMUSART. LNCS, vol. 6025, pp. 311-320. Springer, Istanbul (7-9Apr 2010)

[14] Eysenck, H.J.: An experimental study of aesthetic preference for polygonal figures. The Journal of General Psychology 79(1), 3-17 (1968)

[15] Eysenck, H.J.: The empirical determination of an aesthetic formula. Psychological Review 48(1), 83 (1941)

[16] Eysenck, H.J.: The experimental study of the 'good gestalt' -a new approach. Psychological Review 49(4), 344 (1942)

[17] Franke, H.W.: A cybernetic approach to aesthetics. Leonardo 10(3), 203206 (1977)

[18] Galanter, P.: Computational aesthetic evaluation: past and future. In: McCormack, J., d'IInverno, M. (eds.) Computers and Creativity. pp. 255-293. Springer (2012) 
[19] den Heijer, E.: Autonomous Evolutionary Art. Ph.D. thesis, Vrije Universiteit, Amsterdam (2013)

[20] Jacobsen, T.: Beauty and the brain: culture, history and individual differences in aesthetic appreciation. Journal of anatomy 216(2), 184-191 (2010)

[21] Jacobsen, T., Hofel, L.: Aesthetic judgments of novel graphic patterns: analyses of individual judgments. Perceptual and motor skills 95(3), 755-766 (2002)

[22] Javaheri Javid, M.A., Blackwell, T., Zimmer, R., al Rifaie, M.M.: Spatial Complexity Measure for Characterising Cellular Automata Generated 2D Patterns. In: Pereira, F. and Machado, P. and Costa, E. and Cardoso, A. (ed.) Progress in Artificial Intelligence: 17th Portuguese Conference on Artificial Intelligence, EPIA 2015, Coimbra, Portugal, September 8-11, 2015. Proceedings. Lecture Notes in Artificial Intelligence, vol. 9273, pp. 201-212. Springer International Publishing (2015)

[23] Javaheri Javid, M.A., al Rifaie, M.M., Zimmer, R.: An Informational Model for Cellular Automata Aesthetic Measure. In: AISB 2015 Symposium on Computational Creativity. University of Kent, Canterbury, UK (2015)

[24] Latham, W.H., Todd, S.: Computer sculpture. IBM Systems Journal 28(4), 682-688 (1989)

[25] Li, M.: An introduction to Kolmogorov complexity and its applications. Springer (1997)

[26] Machado, P., Cardoso, A.: Computing aesthetics. In: Advances in Artificial Intelligence, pp. 219-228. Springer (1998)

[27] Machado, P., Romero, J., Manaris, B.: Experiments in computational aesthetics: An iterative approach to stylistic change in evolutionary art. In: Romero, J., Machado, P. (eds.) The Art of Artificial Evolution: A Handbook on Evolutionary Art and Music, pp. 381-415. Springer (2008)

[28] McCormack, J.: Open problems in evolutionary music and art. In: Rothlauf, F., Branke, J., Cagnoni, S., Corne, D.W., Drechsler, R., Jin, Y., Machado, P., Marchiori, E., Romero, J., Smith, G.D., Squillero, G. (eds.) Applications of Evolutionary Computing, EvoWorkshops2005: EvoBIO, EvoCOMNET, EvoHOT, EvoIASP, EvoMUSART, EvoSTOC. LNCS, vol. 3449, pp. 428436. Springer Verlag, Lausanne, Switzerland (30 mar-1 apr 2005)

[29] McCormack, J.: Facing the future: Evolutionary possibilities for humanmachine creativity. In: Romero, J., Machado, P. (eds.) The Art of Artificial Evolution, pp. 417-451. Springer (2008)

[30] Moles, A.: Information theory and esthetic perception. Trans. JE Cohen. U. Illinois Press (1968)

[31] Nake, F.: Information aesthetics: An heroic experiment. Journal of Mathematics and the Arts 6(2-3), 65-75 (2012)

[32] Noll, A.M.: The digital computer as a creative medium. IEEE Spectr. 4(10), 89-95 (Oct 1967)

[33] Rigau, J., Feixas, M., Sbert, M.: Informational aesthetics measures. Computer Graphics and Applications, IEEE 28(2), 24-34 (2008)

[34] Rigau, J., Feixas, M., Sbert, M.: Conceptualizing birkhoff's aesthetic measure using shannon entropy and kolmogorov complexity. In: Cunningham, 
D.W., Meyer, G., Neumann, L. (eds.) Workshop on Computational Aesthetics. pp. 105-112. Eurographics Association, Banff, Alberta, Canada (2007)

[35] Shannon, C.: A Mathematical Theory of Communication. The Bell System Technical Journal 27, 379-423 \& 623-656 (Oct 1948)

[36] Sims, K.: Artificial evolution for computer graphics. Tech. Rep. TR-185, Thinking Machines Corporation (1991)

[37] Staudek, T.: Exact Aesthetics. Object and Scene to Message. Ph.D. thesis, Faculty of Informatics, Masaryk University of Brno (2002)

[38] Wackerbauer, R., Witt, A., Atmanspacher, H., Kurths, J., Scheingraber, H.: A comparative classification of complexity measures. Chaos, Solitons \& Fractals 4(1), 133-173 (1994)

[39] Wilson, D.J.: An experimental investigation of Birkhoff's aesthetic measure. The Journal of Abnormal and Social Psychology 34(3), 390 (July 1939)

[40] Zurek, W.H.: Algorithmic randomness and physical entropy. Physical Review A 40(8), 4731 (1989) 